\title{
Hormonal influence on periodontal tissues during premenstrual and preovulatory periods
}

\section{Chelluboina Meghana1, Sardar Singh Maloth ${ }^{2 *}$, Shyam Sunder Salavadi ${ }^{3}$, Srikanth Chintalapani ${ }^{4}$, Ramesh Babu Mutthineni ${ }^{5}$, Ramanarayana Boyapati ${ }^{6}$}

\begin{abstract}
${ }^{\mathbf{1}}$ Private Practitioner, ${ }^{\mathbf{2}}$ Assistant Professor, ${ }^{\mathbf{3}}$ Associate Professor, ${ }^{4}$ Professor and Head, ${ }^{5}$ Professor, ${ }^{\mathbf{6}}$ Associate Professor, Dept. of Periodontics, ${ }^{1}$ Registered Periodontist, Dental House Centre, Kuwait, ${ }^{2,5}$ Mamata Dental College, Khammam, Telangana, ${ }^{3} \mathrm{Chhattisgarh}$ Dental College and Research Institute, Rajnandgoan, Chhattisgarh, ${ }^{4}$ Malla Reddy Dental College, Hyderabad, Telangana, ${ }^{6}$ Sibar Institute of Dental sciences, Guntur, Telangana, India
\end{abstract}

*Corresponding Author: Sardar Singh Maloth

Email: sardhar3230@gmail.com

\begin{abstract}
The fluctuations in female sex hormones have been shown to affect periodontal health. Sex steroid hormonal levels may lead to increase blood volume, flow rate and vascular permeability in young females. During the menstrual cycle, clinically healthy females demonstrate different periodontal condition. Thus the aim of the present study is to evaluate the influence of hormonal variation on periodontal tissues during premenstrual and preovulatory periods.

Materials and Methods: The sample was composed of 100 women in the reproductive period of 18-35 years. Periodontal parameters i.e bleeding on probing (BOP) and probing depth (PD) were evaluated using calibrated UNC 15 periodontal probe at two different time points, i.e. premenstrual (PM) and preovulatory (PO) periods.

Results: The obtained data was analysed by using t-test. The premenstrual period obtained greater BOP $\{(0.51 \pm 0.11)$ versus $(0.37 \pm 0.08)\}$ and PD $\{(1.82+0.04)$ versus $(1.79 \pm 0.04)\}$ when compared with preovulatory period, showing a statistically significant difference.

Conclusions: Hormonal changes which occur in the women's reproductive period influence periodontal tissues, and in the premenstrual period, there is more susceptibility to gingival inflammation and periodontal disease.
\end{abstract}

Keywords: Gingival status, Female sex hormones, Pre-menstrual and Pre-ovulatory periods.

\section{Introduction}

Periodontitis is an inflammatory disorder that results in the destruction of the structures that support the teeth. This inflammatory process is mainly caused by microbial infections secondary to plaque accumulation. ${ }^{1}$ The response of gingiva to plaque associated periodontal destruction is modified by systemic factors as well, one of them being the changes in hormone levels.

Female reproductive system is a cyclical process in which it involves menstrual period and menopause with fluctuation of estrogen and progesterone secretions as for continued ovulation. ${ }^{2}$ Hormonal fluctuations occur when the anterior pituitary secretes follicle stimulating hormone (FSH) and luteinizing hormone (LH), which result in mature ovary and in the production of estrogen and progesterone. ${ }^{3}$ Numerous studies have described the effect androgenic hormone fluctuations might have on cardiovascular disease $^{[4]}$, urinary tract infection, ${ }^{5,6}$ and immune system. ${ }^{7}$

These steroid hormones may not be the casual agents for periodontal destruction, but they may alter periodontal tissue responses to microbial plaque, and thus indirectly contribute to increased periodontal disease susceptibility. ${ }^{8}$ Periodontal tissues changes such as increased gingival bleeding, probing depth and clinical attachment loss may be explained by the interaction between estrogen and progesterone as chemical mediator of inflammation observed during the periods of hormonal fluctuation. ${ }^{9}$ But data regarding effect of menstrual fluctuations of female sex hormone on the periodontal tissues is scarce.

Thus the purpose of this present longitudinal, prospective study was to evaluate the influence of hormonal variation on periodontal tissues at different time points during their menstrual cycle i.e. premenstrual and preovulatory periods.

\section{Materials and Methods}

This cross-over observational study was conducted on 100 women in the reproductive period during January 2014 to January 2015. The patients were recruited from the department of Periodontics. Study was reviewed by the Institutional Review Board and Ethical committee clearance was obtained prior to study (MDC_R_088093). Informed consent was obtained from participants after explaining the aim of the study.

Patients of age between 18-35 years with regular menstrual cycle and Patients not using contraceptives/other means of hormonal replacement were included in the present study. Pregnant women, patients who were in menopause, metabolic or systemic condition that might affect the periodontium and patients taking immunosuppressive therapy were excluded from the study.

All the patients were divided into two groups and performed periodontal examination:

a) Premenstrual (4 to 5 days before menstruationmaximum peak of progesterone and estrogen).

b) Preovulatory ( 1 to 2 days before ovulation - maximum peak of estrogen; the ovulation occurs in the fourteenth day after the first day of menstruation). ${ }^{3}$

Periodontal examination included was bleeding on probing (BOP) and probing pocket depth (PPD) was evaluated using calibrated UNC-15 periodontal probe.

The BOP, developed by Muhlemann and Son (1971), was used to evaluate the presence of inflammation in periodontal tissues; it was registered 0 bleeding absent on 
dental surface, and 1 for cases in which occur bleeding in any tooth face, evaluating 15 seconds after introducing the millimetre periodontal probe. For measurement, a UNC-15 periodontal probe was introduced inside the gingival sulcus, traversing all of teeth surface (vestibular, palatal/lingual, mesial and distal). ${ }^{10}$

The probing depth (PD) was also recorded with UNC15 periodontal probe. The measurement of depth was the distance from the gingival margins until the position in which the probe has presented resistance. Measures were taken in six points of dental surface (distobuccal, vestibular, mesiovestibular and distolingual, lingual, mesiolingual). These data were recorded in relation to the hormone levels in premenstrual and preovulatory periods.

\section{Results}

All the data obtained in the study were analyzed using SPSS version 14. A p-value of $<0.001$ was considered statistically significant. Comparison of pre-menstrual and pre-ovulatory period was done by using the statistical analysis of independent sample t-student test.

The results shown statistically significant differences in relation to the BOP and PPD of different groups; premenstrual period had higher BOP index $(0.51 \pm 0.11)$ when compared to the preovulatory period $(0.37 \pm 0.08)$. [Table 1].

Table 1: Comparison of gingival bleeding index and probing pocket depth in preovulatory and premenstrual period

\begin{tabular}{|l|c|c|c|c|}
\hline \multirow{2}{*}{} & \multicolumn{2}{|c|}{ Pre-ovulatory } & \multicolumn{2}{c|}{ Pre-Menstrual } \\
\cline { 2 - 5 } & Mean & SD & Mean & SD \\
\hline $\begin{array}{l}\text { Bleeding on } \\
\text { Probing }\end{array}$ & 0.37 & 0.08 & 0.51 & 0.11 \\
\hline $\begin{array}{l}\text { Probing Pocket } \\
\text { Depth }\end{array}$ & 1.79 & 0.04 & 1.82 & 0.04 \\
\hline
\end{tabular}

\section{Discussion}

Epidemiological studies have shown that increased levels of female sex hormones (estrogen and progesterone), has a correlation with an increased prevalence of gingivitis. ${ }^{8}$

The currently accepted periodontal disease classification includes the influence of female sex steroid hormones on the periodontium and significant gingival inflammatory changes have been noted during menstrual cycle. ${ }^{10}$

Estrogen and progesterone hormones have a significant effect on different organ systems in our body. As far as gingiva is concerned, they can influence the cellular proliferation, differentiation and growth of keratinocytes and fibroblasts.

In this cross-over study, the relationship between premenstrual and preovulatory periodontal variables was investigated in 100 patients. Significantly greater mean of BOP and PD were noted in premenstrual period. This may be due to these hormonal i.e. estrogen and progesterone, peak periods are more intense in the premenstrual periods.
Hugoson et al $(1970)^{11}$ in his study reported that more than $75 \%$ of the females exhibit gingival exudate increases at least $20 \%$ during ovulation period. The level of exudate appears to peak just before ovulation, coinciding with the highest levels of female sex hormones in the circulation.

Muhlemann reported a case of "gingivitis intermenstrualis", and he observed bright red haemorrhagic lesions on the interdental papillae which was identified prior to menstruation. Gingival tissues have receptors for female sex hormones and it was reported that these hormones effects on the oral mucosa and the periodontium. ${ }^{12}$ It is also possible that, similar to other organs, vascular endothelial growth factors (VEGF) and VEGF receptors in the gingiva are modulated by estrogen/progesterone, thus contributing to the increase in gingival inflammation in the premenstrual and ovulatory stages of the menstrual cycle. ${ }^{13}$

The effects of female sex hormones on the gingival vasculature could explain the gingival changes observed during pregnancy as well as other stages of the reproductive cycle. An increase in gingival crevicular fluid flow has been correlated to elevated sex hormone levels, which suggests that these hormones may have influence on vascular permeability in the gingival sulcus. ${ }^{10}$

Sex steroid hormones have a minimum effect on clinically healthy periodontium. However, these hormones may exaggerate pre-existing inflammation in periodontal tissues. ${ }^{14}$

Estrogen is the main sex steroid hormone responsible for alterations in blood vessels in target tissues in females stimulating endometrial blood flow during the rise in plasma estrogen seen during the follicular phase. Subsequently, endometrial blood flow decreases during the luteal phase of the cycle with waning estrogen levels. ${ }^{10}$ Progesterone in contrast, has been shown to have little effect on the vasculature of systemic target tissues. Progesterone has been reported to reduce corpuscular flow rate, allowing for accumulation of inflammatory cells. ${ }^{10}$

The results of present study were correlated with the results of other such studies, ${ }^{3}$ in terms of the periodontal status evaluated in patients with premenstrual period and pre ovulatory period.

The limitations of the present study were shorter duration, not recording plaque index, and clinical attachment level. Further research is required to know how sex hormones can influence on periodontal wound healing, and also to examine whether these transitional changes have any lasting negative effects on the Periodontium.

\section{Conclusion}

In light of the above results, it can be concluded that hormones can play a significant role in modulating the periodontal tissue responses and may alter periodontal tissue responses to microbial plaque, and thus directly may contribute to periodontal disease. A better understanding of the periodontal changes to varying hormonal levels throughout life can help the Periodontists in the diagnosis and management. 


\section{Acknowledgements}

Nil

\section{Conflict of interest}

None declared

\section{References}

1. Notuko K, Yoshihiro I, Mamoru K, Ayako O, Aki K, Naoyuki $\mathrm{S}$ et al. Periodic exacerbation of gingival inflammation during the menstrual cycle. J Oral Sci 2005;47:159-64.

2. Ulku B, Ali C, Sevda TK, Alpdogan K, Evin A, Funda Y. Gingival inflammation and interleukin 1-b and tumor necrosis factor-alpha levels in gingival crevicular fluid during the menstrual cycle. J Periodontol 2009;80:1983-90.

3. Souza SP, Martins MV, Molina GO, Pereira J, Oliviera M, Ghizoni J. Influence of hormonal variation on periodontal tissues during premenstrual and preovulatory periods. JRD 2014;2:129-37.

4. Matcheti EE, Mahler D, Sanduri H, Peled M. The effect of menstrual cycle on periodontal health. $J$ Periodontol 2004;75:408-12.

5. Markou E, Eleana B, Lazaros T, Antonios K. The Influence of Sex Steroid Hormones on Gingiva of Women. Open Dent $J$ 2009;3:114-9.

6. Jensen J, Relation between the menstrual cycle and heart disease in women. clinicaltrails.gov 2014.

7. Hextall A. Oestrogens and lower urinary tract function. $J$ Maturitas 2000;36:83-92.

8. Hextall A, Bidmead J, Cardozo L, Hooper R. The impact of the menstrual cycle on urinary symptoms and the results of urodynamic investigation. BJOG 2001;108:1193-96.
9. Pehlivanoglu B, Balkanc ZD, dvanagaoglu AY, Durmazlar N, Ozturk G, Erbas D et al. Impact of Stress, Gender and Menstrual Cycle on Immune System: Possible Role of Nitric Oxide. Arch Physiol Biochem 2001;109:383-87.

10. Guncu GN, Tozum TF, Çaglayan F. Effects of endogenous sex hormones on the periodontium - Review of literature. Aust Dent J 2005;50:138-45.

11. Hugoson A. Gingival inflammation and female sex hormones, a clinical investigation of pregnant women and experimental studies in dogs. J Periodontal Res Suppl 1970;5:6-18.

12. Becerik S, Ozcaka O, Nalbantsoy A, Atilla G, Celec P, Behuliak M et al. Effects of Menstrual Cycle on Periodontal Health and Gingival Crevicular Fluid Markers. J Periodontol 2010;81:673-81.

13. Lindhe J, Branemark PI. Changes in microcirculation after local application of sex hormones. $J$ Periodontal Res 1967;2:185-93.

How to cite this article: Meghana $\mathrm{C}$, Maloth $\mathrm{SS}$, Salavadi SS, Chintalapani S, Mutthineni RB, Boyapati R. Hormonal influence on periodontal tissues during premenstrual and preovulatory periods. Int J Periodontol Implantol 2019;4(3):76-8. 